\title{
Smart cancer nanomedicine
}

\author{
Roy van der Meel $\odot^{1,2,3,4,12}$, Einar Sulheim ${ }^{5,6,7,12}$, Yang Shi ${ }^{8,12}$, Fabian Kiessling ${ }^{8}{ }^{8}$, Willem J. M. Mulder ${ }^{1,2,9}$ \\ and Twan Lammers ${ }^{8,10,11 \star}$
}

\begin{abstract}
Nanomedicines are extensively employed in cancer therapy. We here propose four strategic directions to improve nanomedicine translation and exploitation. (1) Patient stratification has become common practice in oncology drug development. Accordingly, probes and protocols for patient stratification are urgently needed in cancer nanomedicine, to identify individuals suitable for inclusion in clinical trials. (2) Rational drug selection is crucial for clinical and commercial success. Opportunistic choices based on drug availability should be replaced by investments in modular (pro)drug and nanocarrier design. (3) Combination therapies are the mainstay of clinical cancer care. Nanomedicines synergize with pharmacological and physical co-treatments, and should be increasingly integrated in multimodal combination therapy regimens. (4) Immunotherapy is revolutionizing the treatment of cancer. Nanomedicines can modulate the behaviour of myeloid and lymphoid cells, thereby empowering anticancer immunity and immunotherapy efficacy. Alone and especially together, these four directions will fuel and foster the development of successful cancer nanomedicine therapies.
\end{abstract}

$\mathrm{N}$ anomedicine holds potential to improve anticancer therapy ${ }^{1}$. Traditionally, nanomedicines are used to modulate the biodistribution and the target site accumulation of systemically administered chemotherapeutic drugs, thereby improving the balance between their efficacy and toxicity. In preclinical settings, nanomedicines typically increase tumour growth inhibition and prolong survival as compared to non-formulated drugs, but in clinical practice, patients often only benefit from nanomedicines because of reduced or altered side effects ${ }^{2}$.

Despite the recent approval of several nanomedicinal anticancer drugs, such as Onivyde (liposomal irinotecan) and Vyxeos (liposomal daunorubicin plus cytarabine), the success rate of clinical translation remains relatively low. In this context, the striking imbalance between the ever-increasing number of preclinical studies reporting the development of ever more complex nanomedicines on the one hand, and the relatively small number of nanomedicine products approved for clinical use on the other hand, has become the focus of intense debate ${ }^{3,4}$.

Multiple biological, pharmaceutical and translational barriers contribute to this imbalance ${ }^{5}$. Biological barriers include tumour (and metastasis) perfusion, permeability and penetration, as well as delivery to and into target cells, endo/lysosomal escape, and appropriate intracellular processing and trafficking. Pharmaceutical barriers encompass both nanoformulation- and production-associated aspects. These range from a proper stability in the bloodstream, a beneficial biodistribution, an acceptable toxicity profile, and rational mechanisms for drug release, biodegradation and elimination, to issues related to intellectual property position, cost of goods, cost of manufacturing, upscaling and batch-to-batch reproducibility. In terms of clinical translation, the key challenge is to select the right drug and the right combination regimen, and to apply them in the right disease indication and the right patient population.
To make sure that we start tackling the right translational challenges, we must define key strategic directions, to guide nanomedicine clinical trial design and ensure clear therapeutic benefits to patients. In this perspective, we conceptualize 'smart' cancer nanomedicine as an umbrella term for rational and realistic 'Strategies and Materials to Advance and Refine Treatments'. We propose four directions to boost nanomedicine performance and exploitation, that is, smart patient stratification, smart drug selection, smart combination therapies and smart immunomodulation (Fig. 1).

\section{Patient stratification}

Modern oncology drug development extensively employs biomarkers and companion diagnostics for patient stratification. Companion diagnostics help to address the high heterogeneity that is typical of cancer, and they have been instrumental in the successful clinical translation of molecularly targeted drugs, such as growth factor receptor-blocking antibodies and tyrosine kinase inhibitors. As an example, in the trials that led to the approval of Herceptin (trastuzumab) ${ }^{6}$, Perjeta (pertuzumab) ${ }^{7}$ and Kadcyla (ado-trastuzumab emtansine), patients with high human epidermal growth factor receptor 2 (HER2) expression levels were pre-selected via pathological stainings and/or fluorescence in situ hybridization, thereby ensuring enrichment of patients likely to respond and excluding expected non-responders. In immunooncology, the first 'general biomarker', which is not coupled to a particular organ/origin of cancer but instead to a specific genomic signature, has recently been established. This more broadly applicable biomarker is termed microsatellite instability-high (MSI-H) or mismatch repair deficient (dMMR), and it is used for patient stratification in case of treatment with immune checkpoint inhibiting antibodies?.

'Laboratory of Chemical Biology, Department of Biomedical Engineering and Institute for Complex Molecular Systems, Eindhoven University of Technology, Eindhoven, The Netherlands. ${ }^{2}$ Translational and Molecular Imaging Institute, Icahn School of Medicine at Mount Sinai, New York, NY, USA. ${ }^{3}$ Department of Clinical Chemistry and Haematology, University Medical Center Utrecht, Utrecht, The Netherlands. ${ }^{4}$ Department of Biochemistry and Molecular Biology, University of British Columbia, Vancouver, British Columbia, Canada. ${ }^{5}$ Department of Physics, Norwegian University of Science and Technology, Trondheim, Norway. ${ }^{6}$ Department of Biotechnology and Nanomedicine, SINTEF AS, Trondheim, Norway. ${ }^{7}$ Cancer Clinic, St. Olavs University Hospital, Trondheim, Norway. ${ }^{8}$ Institute for Experimental Molecular Imaging, RWTH Aachen University Clinic, Aachen, Germany. ${ }^{9}$ Department of Oncological Sciences, Icahn School of Medicine at Mount Sinai, New York, NY, USA. ${ }^{10}$ Department of Targeted Therapeutics, University of Twente, Enschede, The Netherlands. "'Department of Pharmaceutics, Utrecht University, Utrecht, The Netherlands. ${ }^{12}$ These authors contributed equally: Roy van der Meel, Einar Sulheim and Yang Shi.

*e-mail: tlammers@ukaachen.de 


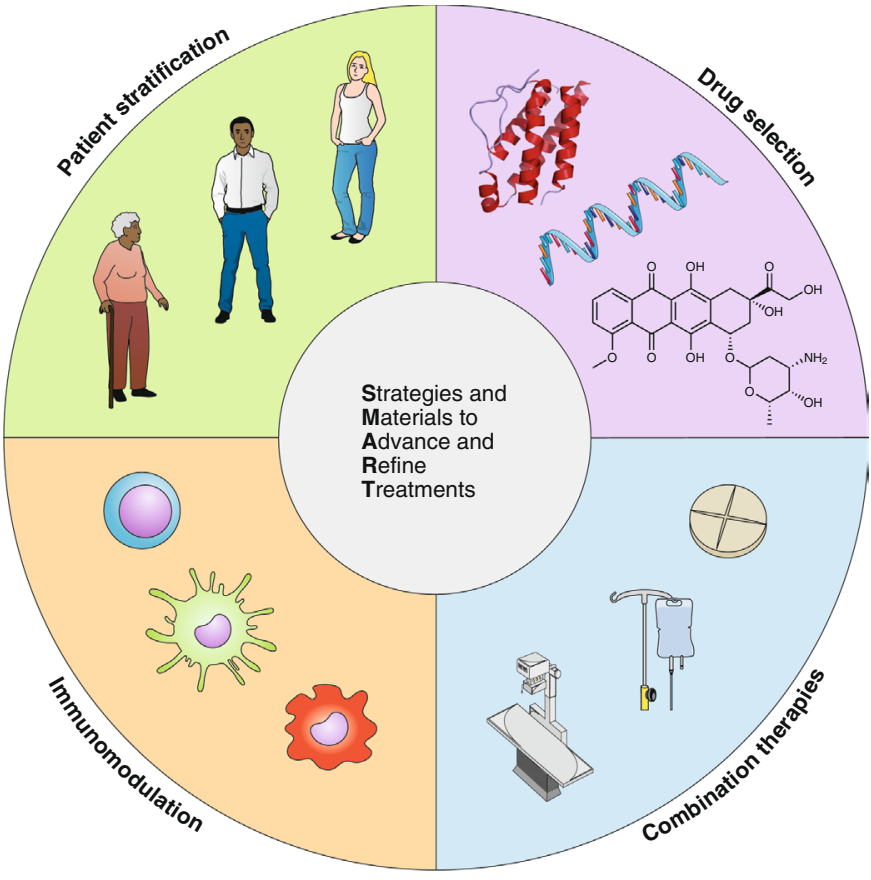

Fig. 1 | Smart strategies and materials to advance and refine cancer nanomedicine treatments. Four directions are proposed that - on their own and especially together - will promote the translation and exploitation of nanomedicinal anticancer drugs.

Biomarkers in cancer nanomedicine. Remarkably, neither biomarkers nor companion diagnostics are currently used to tailor nanomedicine treatments in patients (Fig. 2). Notable exceptions in this regard are antibody-drug conjugates, which are often excluded from nanomedicine lists because they are more biotechnological than nanotechnological (but should be included according to the generally accepted definition). Four antibody-drug conjugates have recently received regulatory approval: Kadcyla (ado-trastuzumab emtansine, anti-HER2); Adcetris (brentuximab vedotin, anti-CD30); Besponsa (inotuzumab ozogamicin, anti-CD22) and Mylotarg (gemtuzumab ozogamicin, anti-CD33). In all of these cases, the intrinsic availability of biomarkers for patient stratification has played a key role in their successful clinical development.

In the case of more traditional cancer nanomedicines, which are based on liposomes, polymeric nanoparticles and micelles, the lack of integrating biomarkers may explain recent failures in the clinic. Notable examples include BIND-014 (docetaxel-loaded prostate-specific membrane antigen (PSMA)-targeted polyethylene glycol-polylactic acid nanoparticles) ${ }^{10}$, CRLX101 (campthotecinloaded polyethylene glycol-cyclodextrin nanoparticles) ${ }^{11}$ and NK105 (paclitaxel-loaded polyethylene glycol-polyaspartate-based micelles) $)^{12}$, which all failed to produce convincing response rates in unstratified patient cohorts. Retrospectively, one can ask whether it was realistic to believe that these nanodrugs could achieve significant response rates in mixed (and heavily pre-treated) patients not stratified in any way. Relatedly, while the US Food and Drug Administration-approved nanomedicine formulations Doxil/ Caelyx and Abraxane have generated substantial patient benefit in thousands of individuals by reducing toxicity and improving quality of life, their therapeutic potential might not yet be optimally exploited, as they are applied in non-stratified patient populations ${ }^{13}$. To improve the clinical impact of cancer nanomedicines, we should therefore start to establish biomarkers for patient stratification, and we should also integrate nanomedicines - ideally already from early clinical development stages onwards - in combination regimens.
Nanomedicine accumulation in solid tumours is generally based on the enhanced permeability and retention (EPR) effect. This concept, however, has come under increasing criticism in recent years. Some nanomedicine investigators even claim that the EPR effect exists only in mice, not in humans. Regardless of whether 'EPR' is pathophysiologically the most correct term to describe the phenomena underlying nanocarrier localization in tumours, passive accumulation definitely occurs in patients but is highly heterogeneous, both inter- and intra-individually. To address this, strategies and materials are needed to monitor and predict nanomedicine accumulation and efficacy ${ }^{14}$. In the case of ligand-targeted nanomedicines, such as BIND-014, which relies on both EPR and active recognition of receptors overexpressed at pathological sites, patient stratification can in principle be achieved relatively easily, for example, via immunohistochemically assessing PSMA expression in tumour biopsies ${ }^{15}$. In addition, PSMA-targeted radiotracers, which are becoming increasingly established in the clinic, can be used to monitor receptor expression on tumour cells and/or angiogenic endothelium via positron emission tomography (PET) or single-photon emission computed tomography (SPECT), allowing for patient stratification also in metastatic patients ${ }^{16}$. Another approach is circulating tumour cell (CTC) analysis, which, when retrospectively applied in a BIND014 phase II clinical trial, indicated that efficient treatment coincided with reduced PSMA-positive CTC $^{10}$. This strategy warrants further investigation, as PSMA-positive (and other) CTC can potentially be employed both as a biomarker for patient stratification and as an indicator of therapeutic responses.

In this context, it is important to keep the basic principles of active tumour targeting in mind. Active targeting relies to a significant extent on passive targeting, which requires prolonged circulation times. Introducing targeting ligands often promotes nanomedicine clearance from the blood stream, via opsonization and accelerated uptake by the mononuclear phagocytic system (MPS) ${ }^{17}$. Before they can bind to target cells, actively targeted nanomedicines furthermore have to extravasate out of the blood vessels in tumours and metastases, penetrate deep into the interstitium and cross multiple cell layers. The latter can suffer from the so called binding-site barrier ${ }^{18}$, which may hinder nanomedicine penetration ${ }^{19}$. Nanocarrier size plays a crucial role in determining the added value of active targeting. It has for instance been shown that small-sized $(\sim 7 \mathrm{~nm})$ polymeric nanocarriers achieve much higher levels of tumour accumulation if they are actively targeted, because of improved retention ${ }^{20}$. Conversely, for larger $(\sim 14 \mathrm{~nm})$ nanocarriers, no difference in tumour accumulation was observed between passive and active targeting. Active targeting did, however, change the intratumoural compartmentalization of the nanocarriers, increasing their uptake by cancer cells. In line with this, it was shown that 70-80 nm-sized HER2-targeted gold nanoparticles ${ }^{21}$ and $100 \mathrm{~nm}$-sized HER2-targeted liposomes ${ }^{22}$ accumulate to a higher extent in cancer cells than in macrophages, but do not achieve higher tumour concentrations. These scenarios underline the importance of critically reflecting on the added value of active targeting in cancer nanomedicine applications. In our view, actively targeted nanomedicines will be mainly beneficial for the treatment of haematological cancers (in which significant numbers of cancer cells are present in systemic circulation), in set-ups in which certain immune cells are targeted (in the blood stream and/or in MPS organs), and when the therapeutic payload needs to be delivered intracellularly (for example, in the case of RNA drugs employed to modulate gene expression).

Imaging biomarkers. The use of liquid and tissue biopsy biomarkers is less straightforward in the case of passively targeted nanomedicines, as there are no surface receptors available for immunohistochemical staining or CTC assessment. Accordingly, these nanomedicines likely require imaging probes and protocols for patient stratification (Fig. 2). Several recent studies have 

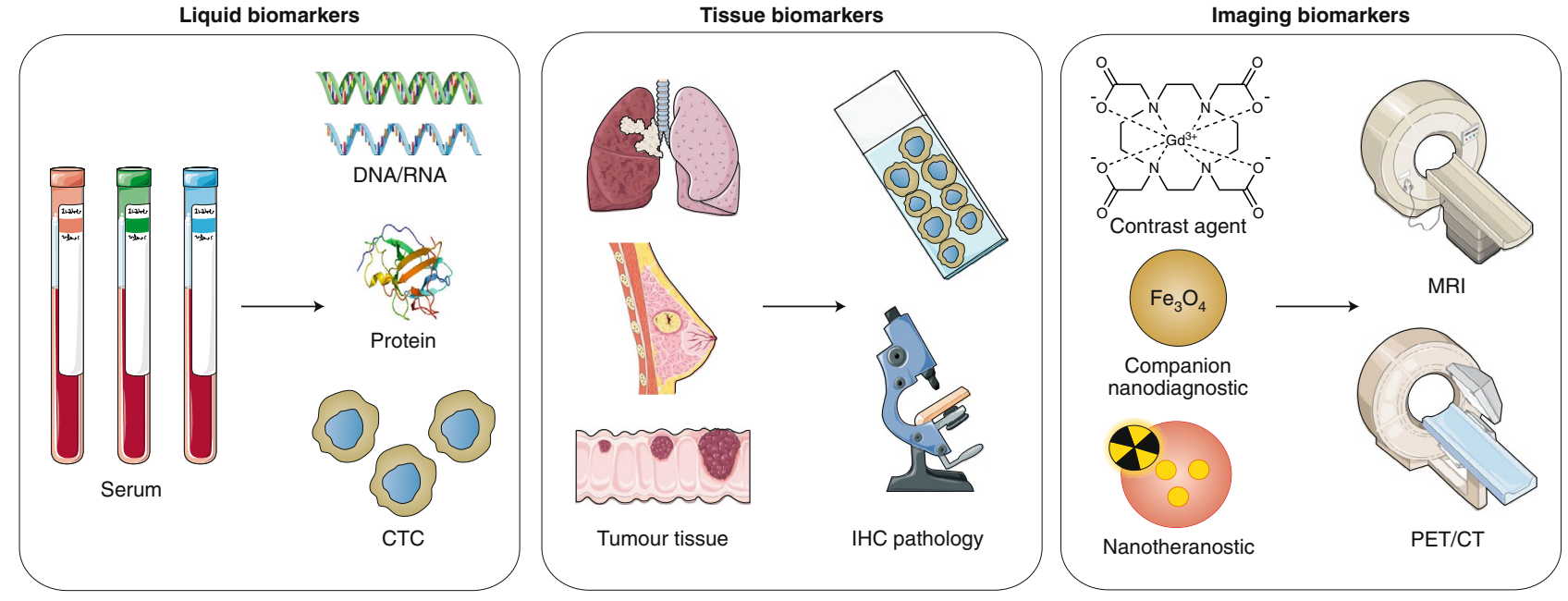

Fig. 2 | Smart strategies for patient stratification in cancer nanomedicine. Several probes and protocols can be employed for patient stratification, including circulating tumour cell (CTC) analysis, immunohistochemical (IHC) assessment of the tumour microenvironment, and direct and indirect imaging of nanomedicine tumour accumulation. These approaches vary in simplicity, specificity and applicability for passively versus actively targeted nanomedicines. Liquid biomarkers are the most straightforward and least invasive, but may not be predictive enough to serve as standalone biomarkers for tailoring nanomedicine treatment. Tissue biomarkers are easily available, but are likely more useful for actively than passively targeted nanomedicines, and their predictive power needs to be explored. Imaging biomarkers can rely on approved contrast agents and companion nanodiagnostics, which are available off the shelf. This contributes to simplicity, but the information obtained may not be specific enough. Nanotheranostics provide highly specific information on the target site accumulation of the formulation in question, but are more challenging from a translational point of view. MRI, magnetic resonance imaging; $\mathrm{PET} / \mathrm{CT}$, positron emission tomography/computed tomography.

established companion nanodiagnostics and nanotheranostics to address EPR effect heterogeneity and to predict nanotherapy outcomes $^{23,24}$. For example, ferumoxytol (that is, FDA-approved $30 \mathrm{~nm}$ iron oxide nanoparticles used to treat iron deficiency anaemia) can be used 'off-label' to characterize EPR heterogeneity via magnetic resonance imaging $(\mathrm{MRI})^{25}$. Ferumoxytol-enhanced MRI correlated with therapeutic nanoparticle uptake in tumourassociated macrophages (TAM) and enabled prediction of tumour accumulation and anti-tumour efficacy ${ }^{25}$. In the clinic, ferumoxytolenhanced MRI in patients with mixed solid tumours demonstrated that higher ferumoxytol accumulation levels correlated with greater lesion size reductions following treatment with liposomal irinotecan (Onivyde) ${ }^{26}$. Although such MRI-based companion diagnostics are relatively cost effective, they require pre- and post-contrast MRI, which complicates the analysis, particularly in regions with variable soft tissue contrast. An alternative companion nanodiagnostic approach is positron emission tomography (PET) imaging with ${ }^{89} \mathrm{Zr}$-labelled nanoreporter liposomes, which accurately predicted Doxil accumulation and efficacy using a single PET scan ${ }^{27}$. Besides predicting tumour accumulation, imaging biomarkers can also be employed to assess responses to nanomedicine therapies. Tumour metabolism - as opposed to that in healthy cells - largely relies on aerobic glycolysis, a phenomenon known as the Warburg effect ${ }^{28}$. This phenomenon is clinically routinely visualized and quantified using $18 \mathrm{~F}$-fluoro-2-deoxyglucose (18F-FDG)-based PET imaging ${ }^{29}$. 18F-FDG PET imaging can be employed to assess early responses to nanomedicine therapies, for example, to nano-photothermal therapy ${ }^{30}$. A related and highly interesting future direction in this regard is imaging and targeting of amino acid metabolism in tumours. Particularly glutamine metabolism $^{31,32}$, but also the availability and consumption of aspartate $^{33}$ and asparagine ${ }^{34}$ have recently been shown to be involved in tumour growth and/or metastasis. Nanomedicine formulations interfering with these pathways and imaging agents capturing these pathways are envisaged to hold significant potential for improving cancer therapy.

Cancer nanomedicines can in principle be co-loaded with drugs and with imaging agents, to directly visualize and quantify target site accumulation. Such theranostic formulations provide the most specific information on biodistribution and tumour accumulation, thereby ruling out issues related to differences in physicochemical and pharmacokinetic properties between nanodiagnostics and nanotherapeutics. However, these formulations are more difficult to translate to the clinic and lack the flexibility and versatility of companion nanodiagnostics, which allow for example, for decision making prior to starting treatment. A pioneering clinical study involving nanotheranostics recently showed that PET combined with computed tomography $(\mathrm{CT})$ can assess the tumour accumulation of ${ }^{64} \mathrm{Cu}$-labelled HER2-targeted liposomal doxorubicin in HER2-positive metastatic breast cancer patients. Higher target site accumulation levels corresponded with more favourable therapeutic outcomes ${ }^{35}$. Interestingly, molecules such as doxorubicin and alendronate can function as chelators, to enable easy and efficient in vivo monitoring of target site accumulation ${ }^{36}$. This smart and straightforward approach to obtain radiolabelled liposomal drugs opens up new theranostic opportunities for patient stratification in cancer nanomedicine.

It has to be kept in mind, however, that nanomedicine accumulation in tumours must be followed by payload release to achieve therapeutic benefits, since for most nanomedicines, the formulated drugs have to be liberated to become pharmacologically active. Drugs can be spontaneously released from nanocarriers in tumours, for example free doxorubicin is released from Doxil ${ }^{37}$, and this can also be mediated by $\mathrm{TAM}^{38}$. Monitoring drug release in vivo is possible using contrast-enhanced MRI and optical imaging. Both methods, however, have limited translational relevance, because of the potential toxicity associated with co-loading high amounts of gadolinium in nanomedicines, and because of the limited penetration depth of optical imaging, respectively. Consequently, 
studies in two-dimensional (2D) cell culture, in 3D spheroids and in preclinical mouse models should encompass mechanistic analyses on drug release and target cell uptake upon nanomedicinemediated delivery.

As an alternative to companion nanodiagnostics and nanotheranostics, clinically established (but intuitively significantly less specific) 'standard' imaging protocols could be considered to predict the accumulation and efficacy of passively targeted cancer nanomedicines, such as dynamic contrast-enhanced $\mathrm{MRI}^{39}, \mathrm{CT}^{40}$ and ultrasound ${ }^{41}$. Furthermore, future analyses should set out to evaluate if histopathological assessment of tumour biopsies can help to stratify responders from non-responders. Tumour biopsies are readily available for the vast majority of patients, and scoring, for example, vessel and macrophage density and distribution is hypothesized to be reasonably useful for predicting nanomedicine accumulation and efficacy.

\section{Drug selection}

Rational drug selection is crucial for making cancer nanomedicines clinically and commercially successful. Preclinically, most nanomedicine publications deal with re-formulating established chemotherapeutic drugs, and all clinically approved cancer nanomedicines (antibody-drug conjugates excluded) are based on standard cytostatics, such as doxorubicin, daunorubicin, paclitaxel, vincristine and irinotecan ${ }^{42}$. For all of these agents, nanomedicine re-formulation improves the therapeutic index, but typically mostly by attenuating side effects, not by inducing significantly improved therapeutic responses. In this context, specific nanomedicine-related side effects, such as complement-mediated infusion reactions ${ }^{43}$, have to be kept in mind, and a better mechanistic understanding of these effects, and the identification of efficient ways of dealing with them, is crucial for maximizing nanomedicines' impact on the therapeutic index ${ }^{5}$. In addition, future efforts in cancer nanomedicine drug development should focus more on the use of nanocarrier materials for the delivery of non-standard drugs such as biologics, and they should increasingly encompass smart strategies such as drug derivatization, modular nanocarrier design and library screening (Fig. 3).

Drug classes. Standard chemotherapeutic drugs, such as doxorubicin and paclitaxel, do come with side effects, but are overall reasonably well tolerated by patients; otherwise they would not have become drug products. Newer, more potent, agents, such as auristatins, are too toxic to be administered to patients in free form. To enable the in vivo use of auristatins, antibody-drug conjugates have been developed, such as brentuximab vedotin (Adcetris), which is approved to treat $\mathrm{CD} 30^{+}$haematological cancers. Antibody-drug conjugates, however, typically have very low payloads. To explore formulations with higher auristatin payloads, polymeric nanoparticles containing thousands of drug molecules were developed. These nanoparticles achieved efficient tumour growth inhibition and prolonged survival as compared to standard of care (cisplatin) in a patient-derived xenograft model of ovarian cancer, without significant systemic toxicity ${ }^{44}$. Similarly, a potent Aurora B kinase inhibitor - which in free form caused unacceptable side effects in a phase II clinical trial - increased efficacy and reduced toxicity in multiple preclinical models upon reformulation in PEG-PLA-based nanoparticles ${ }^{45}$.

Nanocarrier technologies also play a prominent role in the development of DNA- and RNA-based drugs. Nucleic acid agents critically rely on protection against degradation in systemic circulation and they generally require intracellular delivery. The nanotechnology used in Onpattro (patisiran, liver-targeted siRNA-containing lipid nanoparticles for the treatment of transthyretin-related hereditary amyloidosis ${ }^{46}$ ) is facilitating the development of other genetic nanomedicines, such as RNA-based vaccines for treating infections and cancer ${ }^{47,48}$. These vaccines contain RNA encoding for therapeutic proteins and upon delivery to hepatocytes, the liver is utilized as a bio-factory for protein production ${ }^{49}$. Similarly, nanoparticles carrying RNA encoding for tumour antigens can be delivered to antigen-presenting cells in the liver and spleen to elicit $\mathrm{T}$ cell-mediated anti-tumour effects ${ }^{50-52}$. Several such nucleic acidbased nanovaccines have recently entered clinical trials for cancer immunotherapy ${ }^{47,50,53}$.

Proteins are another class of therapeutics that benefit from nanocarrier delivery, for instance by abrogating the systemic toxicity of immunostimulatory agents such as interleukin-2 (IL2) for immunooncology applications ${ }^{54}$. NKTR-214 (bempegaldesleukin), that is, a biologic prodrug comprising IL2 with 6 conjugated releasable PEG chains, significantly inhibited tumour growth in a melanoma mouse model compared to unmodified IL2 (aldesleukin) and it synergized with checkpoint inhibitor therapy ${ }^{55}$. NKTR-214 treatment was furthermore found to be well tolerated in non-human primates ${ }^{55}$ and in patients with advanced or metastatic solid tumours ${ }^{56}$.

Modular design. Nanomedicine design has traditionally been formulation driven; for every new nanodrug, the carrier material and formulation process need to be adjusted to the payload's physicochemical properties. While this approach is inherently inflexible, it can be applied to therapeutics with similar characteristics. For example, $\mathrm{pH}$ gradient-based remote loading has been widely employed to entrap amphiphilic and ionizable drugs, such as doxorubicin, in liposomes ${ }^{57}$. As an alternative, drug molecules can be (re-)engineered to improve their compatibility with nanocarriers. For example, rational chemical derivatization of doxorubicin modified the drug's hydrophobicity and miscibility with PLGA nanoparticles to improve drug-nanocarrier compatibility and therapeutic efficacy ${ }^{58}$. Analogously, a hydrolysable ester linker conjugated to docetaxel enabled stable incorporation in core-crosslinked polymeric micelles while still allowing for controlled drug release. A single intravenous injection of polymeric micelles loaded with the docetaxel derivative induced complete tumour regression in a xenograft model of breast cancer $^{59}$. This platform is currently being evaluated in a phase II trial for treatment of ovarian cancer ${ }^{60}$. Using a similar ester linker technology, fatty acids conjugated to cabazitaxel inducted prodrugs and PEG-lipids to self-assemble into nanoparticles. The resulting PEGylated cabazitaxel-prodrug had reduced systemic toxicity and superior therapeutic efficacy when compared to free cabazitaxel in mice bearing breast cancer xenografts ${ }^{61}$.

Drug derivatization techniques are increasingly used to develop modular nanomedicine platforms. Synthesizing prodrugs that stably incorporate in nanocarriers reduces differences among drug molecules' physicochemical properties, thereby improving the encapsulation predictability of drugs with diverse characteristics. In addition, modular prodrug nanomedicines can be employed to control localized drug release, which reduces systemic exposure and side effects. For example, a smart prodrug-based modular nanomedicine platform stably co-encapsulated doxorubicin and monomethyl auristatin $\mathrm{E}$ (which are physicochemically very different), and allowed for prodrug activation via the administration of a separate nanoparticle, together achieving improved selectivity and treatment efficacy in fibrosarcoma-bearing mice ${ }^{62}$.

Screening nanomedicine libraries. Drug derivatization and modular concepts combined with rapid and scalable production methods have been expediting the formation of nanomedicine libraries for candidate selection during preclinical nanodrug development. Accordingly, efficient in vitro and in vivo screening methods need to be established to evaluate such libraries. Traditional approaches only vary a few parameters, such as size and surface charge, when exploring a new nanomedicine's pharmaceutical properties and pharmacological performance. Novel production methods, such 


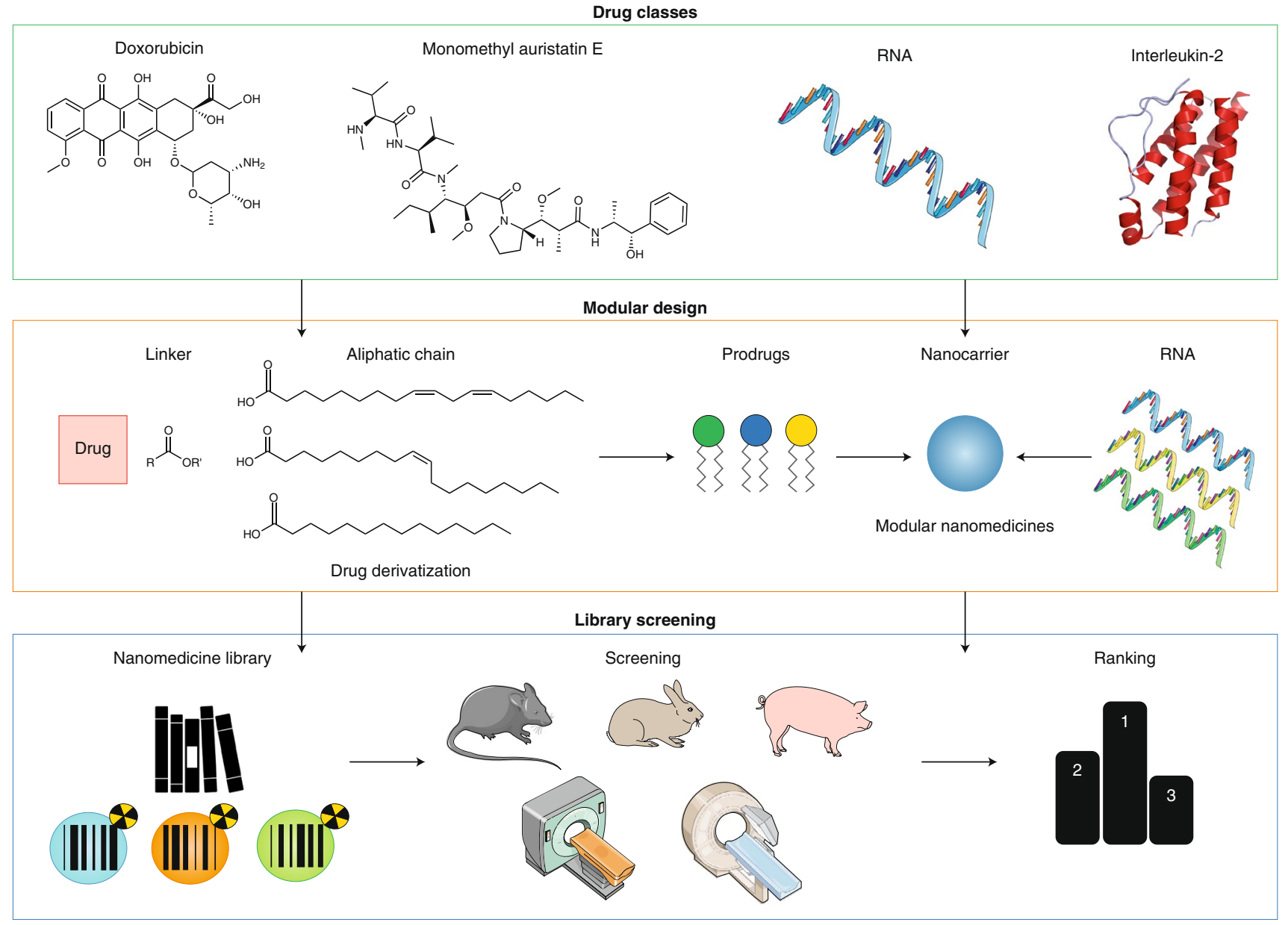

Fig. 3 | Smart drug selection in cancer nanomedicine. Rational drug selection is crucial to ensure clinical and commercial success. Multiple strategies can be envisaged to connect the right drug to the right nanocarrier for the right indication. Drug classes: Nanomedicines can be loaded with different types of anticancer drugs, including standard chemotherapeutics, highly potent toxins, biologics and nucleic acids. Prodrugs can be engineered to ensure optimal compatibility with nanocarrier formulations, including, for example, drug coupling via ester linkers to an aliphatic chain for efficient incorporation in lipidbased nanomedicines. Modularity: Nanomedicines can be designed to encapsulate payloads with comparable/compatible properties, such as RNA or small molecule prodrugs. Screening: Nanomedicine libraries can be produced via high-throughput technologies. Various labelling and analytical techniques can be employed to identify nanomedicine candidates with optimal properties for in vivo performance.

as microfluidics, facilitate the generation of large nanomedicinal libraries and thereby promote systematic screening of multiple parameters. Such microfluidic production protocols were employed in the development of BIND-014 (that is, PSMA-targeted polymeric nanoparticles containing docetaxel $)^{63}$. To optimize BIND014, a combinatorial polymeric nanoparticle library was formed and subsequently screened for nanoparticle size, ligand density, drug encapsulation and release kinetics. Formulations with the most favourable characteristics were taken forward for pharmacokinetic, biodistribution, tolerability and therapeutic efficacy studies in animal models, and the most optimal formulation was eventually tested in patients ${ }^{63}$.

In pursuit of innovative nano-immunotherapeutics, PET imaging and flow cytometry were used to evaluate the biodistribution and immune cell specificity of a library of high-density lipoproteinmimicking nanoparticles. To advance cancer vaccine development, libraries of lipid nanoparticles containing mRNA encoding for the model protein ovalbumin ${ }^{52}$ were screened for the percentages of ovalbumin-specific $\mathrm{CD}^{+} \mathrm{T}$ cells 7 days after subcutaneous injection. After a two-step library selection to optimize lipid components and molar composition, the formulation that induced the highest number of specific $\mathrm{CD}^{+} \mathrm{T}$ cells was loaded with mRNA encoding for tumour-associated antigens, resulting in improved survival of mice bearing B16-F10 melanoma tumours ${ }^{52}$. Barcoding methods are useful in library screening. Deep sequencing of nucleic acidbased barcodes in tissues upon the intravenous administration of 30 barcoded lipid nanoparticles helped to identify a nanoparticle formulation capable of delivering functional siRNA and sgRNA to bone marrow endothelial cells ${ }^{64,65}$. Another smart barcoding approach involved the co-loading of different chemotherapeutics together with DNA barcodes in PEGylated liposomes that were administered to mice, followed by tumour biopsy $48 \mathrm{~h}$ later. PCR barcode detection and cell viability assessment in isolated tumour cells could predict the most effective chemotherapeutic treatment in a mouse model of breast cancer ${ }^{66}$.

\section{Combination therapies}

Depending on their location, grade and stage, cancers are treated with a combination of surgery, radiotherapy, chemotherapy and/ or immunotherapy. Strikingly, however, clinical trials involving nanomedicines are generally designed for evaluation in monotherapy settings. This makes it difficult to compare nanodrugs to standard of care in terms of efficacy and, more importantly, it does not optimally exploit some of the intrinsic capabilities of 


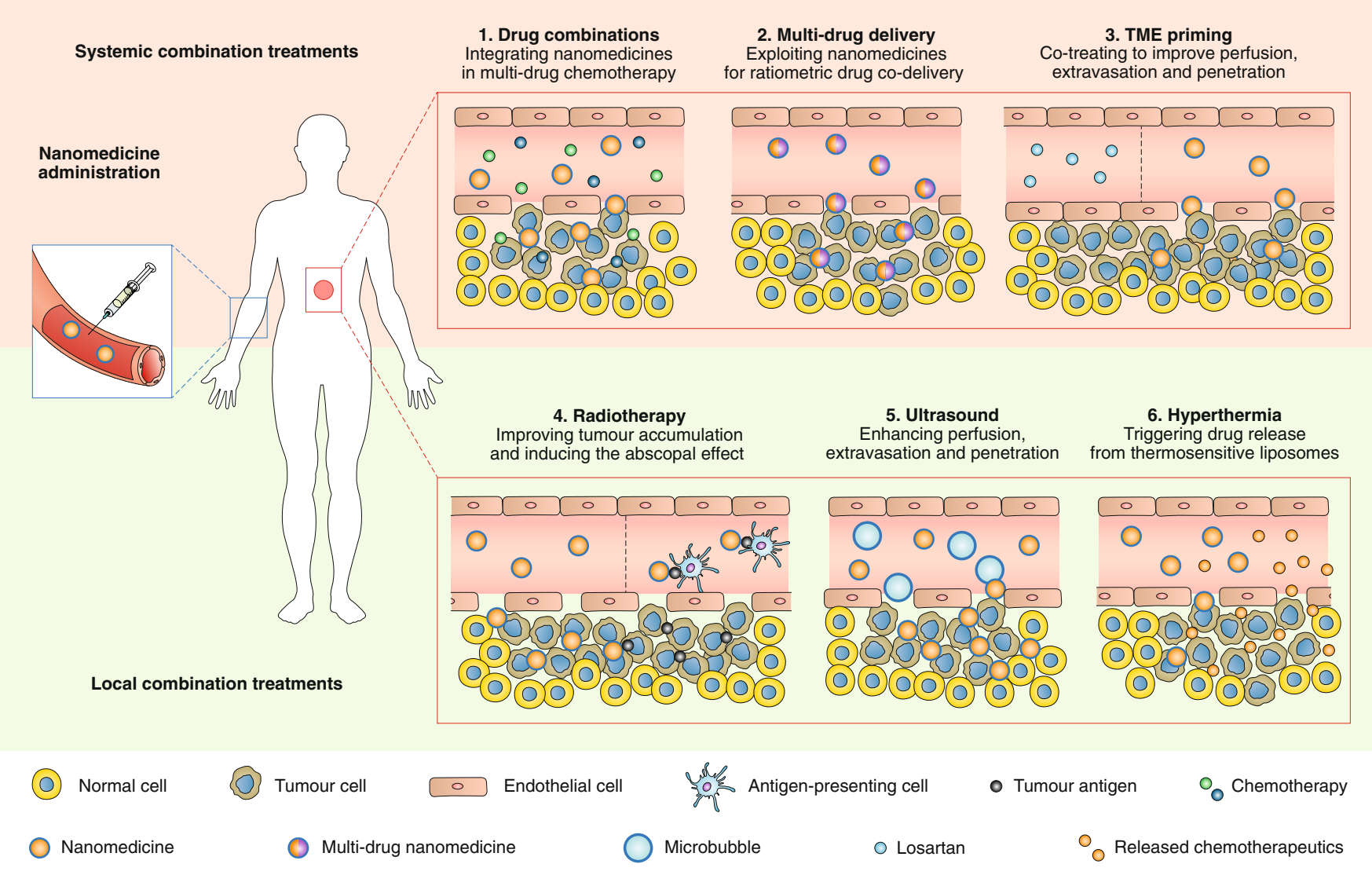

Fig. 4 | Smart cancer nanomedicine-based combination therapies. Systemic combinations: (1) Integrating nanomedicines in multimodal chemotherapy regimens results in reduced side effects, better patient compliance and improved quality of life; (2) Nanomedicines enable ratiometric multi-drug delivery, which contributes to synergistic drug effects by improving control over pharmacokinetic and pharmacodynamic interactions; (3) Co-treatment with approved drugs, such as losartan, helps prime blood vessels and the tumour microenvironment (TME) for improved delivery, thereby enhancing the accumulation, penetration and efficacy of cancer nanomedicines. Local combination: (4) Radiotherapy treatment alters the TME to improve the accumulation, penetration, retention and efficacy of cancer nanomedicines. In addition, nanomedicines can potentiate the abscopal effect upon radiotherapy; (5) Ultrasound and microbubbles can induce sonopermeation, thereby increasing vascular perfusion and permeability, and nanomedicine accumulation and efficacy; (6) Hyperthermia can be used to locally trigger payload release from temperature-sensitive liposomes, resulting in increased drug concentrations at the pathological site and less systemic drug exposure, thereby improving the balance between drug efficacy and toxicity.

nanomedicine formulations. Indeed, nanomedicines combine well with standard chemotherapy treatments, they can co-encapsulate multiple drugs in one formulation and they interact with external stimuli more effectively than drugs in free form. Regarding the latter, (1) nanomedicines depend more on vascular perfusion and permeability than small molecule drugs, and these features profit from using pharmacological and physical combination therapies; (2) nanomedicines deliver higher amounts of drugs to pathological sites, and keep them there for prolonged periods of time, thereby beneficially affecting locally applied physical combination therapies; and (3) nanomedicines can be designed to specifically respond to locally applied physical triggers, for example, via inducing drug release. These aspects should be exploited more extensively to create more effective anticancer combination therapies (Fig. 4).

Systemic combination therapies. The value of incorporating nanomedicines in systemic combination therapies is exemplified by liposomal irinotecan (Onivyde), which has recently been approved in combination with 5-fluorouracil (5-FU) and leucovorin (LV) to treat pancreatic cancer. Although irinotecan is a very potent anticancer drug, its application in free form is hampered by severe side effects that lead to poor patient compliance. While liposomal irinotecan alone did not improve overall survival as compared to
5-FU/LV in pancreatic cancer patients, the combination of liposomal irinotecan and 5-FU/LV significantly improved overall survival with very acceptable toxicity profiles ${ }^{67,68}$. A similarly promising systemic combination therapy is nanoparticle albumin-bound paclitaxel (Abraxane) combined with the immune checkpoint inhibitor atezolizumab, which together induced unprecedented therapeutic responses in triple-negative breast cancer patients ${ }^{69}$.

Cancer nanomedicines are increasingly combined with systemically administered drugs that help overcome barriers associated with tumour-targeted drug delivery. Multiple biological, immunological and translational barriers have been identified that need to be tackled when aiming to increase cancer nanomedicine's clinical impact and performance ${ }^{5}$. Biological barriers include the dense extracellular matrix (ECM), the high interstitial fluid pressure (IFP) and the high metabolism in tumours. The angiotensin II receptor inhibitor losartan can be used to inhibit TGF- $\beta$ signalling and attenuate collagen production by cancer-associated fibroblasts, thereby reducing the ECM content and the IFP in tumours, and improving the delivery and efficacy of both conventional nanomedicines and $100-\mathrm{nm}$-sized oncolytic viruses ${ }^{70,71}$. Recently, losartan has been shown to substantially increase the fraction of R0 resections upon FOLFIRINOX-based neoadjuvant chemotherapy in pancreatic cancer, likely via alleviating the impact of some of the above biological 
barriers $^{72,73}$. The enhanced cellular metabolism within tumours results in a relatively low extracellular $\mathrm{pH}(\mathrm{pH}$ 6.5-7) and re-adjusting this metabolic phenotype can help to promote the performance of drug and drug delivery systems. As an example, in a recent study, 100 -nm liposomes loaded with sodium bicarbonate were shown to be able to increase the intratumoural $\mathrm{pH}$ to $\sim 7.4$, thereby enhancing the cellular uptake of doxorubicin via preventing its protonation and hydrophilization ${ }^{74}$.

Nanomedicines are also intrinsically very useful for systemic combination therapy. For example, Vyxeos is a liposomal nanomedicine formulation co-loaded with cytarabine and daunorubicin at a synergistic drug ratio of 5:1, ensuring controllable pharmacokinetics and co-delivery for both entrapped agents, that can be used to 'ratiometrically' kill cancer cells. In a phase III trial in patients with acute myeloid leukaemia, Vyxeos improved survival as compared to standard of care from 6 to 10 months $s^{75}$, resulting in fast-track FDA approval.

Local combination therapies. Cancer nanomedicines combine well with locally confined treatment modalities, such as radiotherapy, ultrasound and hyperthermia. Pre-treating tumours with radiotherapy increases nanomedicine accumulation and penetration, and vice versa, nanocarrier materials improve radiochemotherapy outcomes $^{76,77}$. Radiotherapy combined with cyclophosphamide can prime tumours for more efficient delivery and efficacy of liposomal irinotecan ${ }^{78}$. In addition, nanomedicines can capture tumour antigens released by radiotherapy and subsequently deliver them to antigen-presenting cells, resulting in significantly improved immunotherapy outcomes via promotion of the abscopal effect ${ }^{79}$.

Ultrasound can be combined with microbubbles to enhance tumour-targeted drug delivery via sonopermeation ${ }^{80}$, the effects of which include opening inter-endothelial junctions, promoting endocytosis and transcytosis, and - particularly in highly stromal tumours - improving vascular perfusion ${ }^{5}$. Initial clinical proof of concept for sonopermeation showed that combining gemcitabine with ultrasound and microbubbles in pancreatic cancer patients resulted in a median survival time of 18 months, as compared to 9 months for a historical cohort treated with gemcitabine alone ${ }^{81}$. When combined with intravenously administered microbubbles, a $1 \mathrm{~cm}$-sized skull-implantable ultrasound device termed SonoCloud can open up the blood-brain barrier (BBB), promoting carboplatin delivery in patients with glioblastoma ${ }^{82}$. For externally applied ultrasound, magnetic resonance-guided focused ultrasound can feasibly and safely improve (nano-)drug delivery in patients with primary brain tumours ${ }^{83}$.

The clinically most advanced approach combining nanomedicines with locally applied physical treatments involves the use of temperature-sensitive doxorubicin-loaded liposomes (Thermodox). In combination with radiofrequency ablation-based hyperthermia, Thermodox did not meet its primary endpoint in a phase III trial for treatment of hepatocellular carcinoma (HCC), for a number of reasons. It did however demonstrate improved progression-free and overall survival in a subset of patients ${ }^{84}$. A follow-up phase III trial in HCC, with rigorously refined RFA settings, has recently been completed, and the outcomes of this study are eagerly awaited ${ }^{85}$. Furthermore, in the last couple of years, several additional trials have recently been initiated in which Thermodox is combined with focused ultrasound-based hyperthermia, for example, for breast cancer and certain paediatric tumours ${ }^{80,86}$.

\section{Immunomodulation}

Immune checkpoint inhibitors ${ }^{87}$ and chimeric antigen receptor (CAR) $\mathrm{T}$ cell therapies ${ }^{88}$ are radically changing the cancer therapy landscape, as evidenced by remarkable recent clinical results. Empowering the body's immune system has produced long-lasting anti-tumour responses in patients with cancer types and stages that were deemed very difficult or impossible to treat. Most impressively, long-term follow-up studies show that a subset of patients have experienced complete remission thus far for up to a decade. Unfortunately, however, the majority of cancer patients do not respond to immunotherapy. To improve immunotherapy outcomes, we have to expand our understanding of both systemic anti-tumour immunity and the local tumour immune microenvironment (TIME) ${ }^{89}$. This knowledge will guide the development of drugs and drug delivery systems that boost the efficacy of immunooncological interventions ${ }^{5}$.

Since its inception in the late 1990s, cancer nanomedicine development has been based on designing drug delivery systems that evade the immune system and transport therapeutic payloads directly to tumour cells. We now understand that nanomedicines' true potential may actually lie in their ability to engage the immune system. Deploying nanotherapeutics for immuno-oncological purposes $^{90-92}$ necessitates nanoformulations that can smartly modulate the adaptive and/or the innate immune system (Fig. 5). We anticipate it will soon become feasible to engineer nanomedicines that boost the immune system's ability to fight cancer and/or improve the efficacy of existing immunotherapies while mitigating some of their side effects, which can be quite severe ${ }^{93,94}$.

Targeting the adaptive immune system. In line with currently approved anticancer immunotherapeutics, most immunomodulating nanomedicines target the adaptive immune system. These formulations are generally designed to elicit anti-tumour effects via generating $\mathrm{CD}^{+}{ }^{+}$and $\mathrm{CD} 8^{+} \mathrm{T}$ cell responses, inducing or improving antigen presentation (signal 1), modulating co-stimulatory signals (signal 2) and/or triggering cytokine production (signal 3). In addition, immunomodulating nanomedicines can be devised to alter the TIME and thereby increase susceptibility to immunotherapy.

Nanomedicines that deliver chemotherapeutics such as doxorubicin and oxaliplatin to tumours can promote anti-tumour immunity by inducing immunogenic cell death (ICD), thereby potentiating the effects of checkpoint blockade immunotherapeutics ${ }^{95}$. A key reason for why ICD-inducing nanomedicines generate better immunotherapy outcomes than ICD-inducing free drugs is that systemic drug exposure is reduced in case of the former, resulting in less systemic immunodepression. Nanomedicines can also curtail the activity of soluble immuno-inhibitors, like indoleamine 2,3-dioxygenase (IDO). IDO plays a key role in cancer immune metabolism, promoting the conversion of tryptophan to kynurenine, thereby inducing immunosuppression ${ }^{96}$. Nanomedicines containing IDO inhibitors inactivate this immunosuppressive metabolic pathway. As an example, nanoparticles containing oxaliplatin and a small molecule IDO inhibitor induced regression in pancreatic ductal adenocarcinoma mouse models following both local and systemic administration ${ }^{97}$. Similarly, in a mouse model of metastatic breast cancer, the therapeutic efficacy of anti-PD-1 therapy was improved by a nano-coformulation of doxorubicin and an IDO inhibitor ${ }^{98}$.

As described in the 'Drug selection' section, developing personalized cancer vaccines is the most clinically advanced genetic drug application in oncology. These vaccines consist of an ionizable lipid-based carrier system loaded with mRNA encoding for patient-specific tumour antigens $\mathrm{s}^{50-52}$. In such set-ups, the nanocarrier formulation protects the payload following subcutaneous $s^{52}$ or intravenous $^{50,51}$ administration, thereby ensuring efficient antigen delivery to antigen-presenting cells and subsequently inducing effective anti-tumour $\mathrm{T}$ cell responses. The interchangeable RNA payloads can be designed to encode either for tumour-associated self-antigens shared by patients with a specific tumour type or for patient-specific neoepitopes, thus creating truly personalized cancer vaccines ${ }^{47}$.

Analogous to some of the results described in the 'Combination therapies' section, studies show that under certain circumstances, 


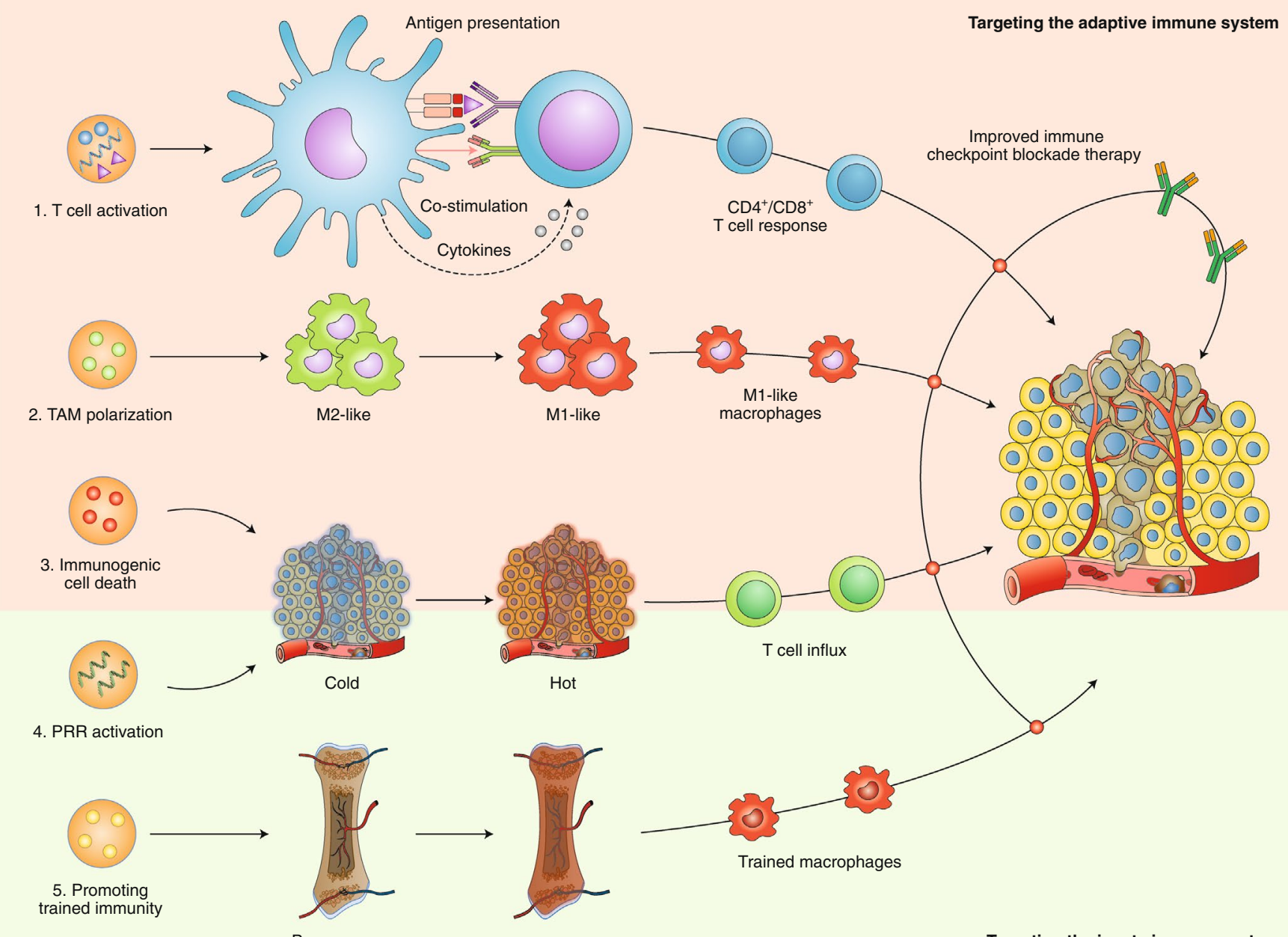

Bone marrow

Targeting the innate immune system

Fig. 5 | Smart immunomodulation involving cancer nanomedicine. Nanomedicines can be designed to target and modulate components of the adaptive and innate immune systems, thereby improving the outcome of immune checkpoint inhibition therapy. Adaptive immune system-targeted approaches include: (1) Directly targeting antigen-presenting cells by using nanomedicines to deliver (RNA encoding for) tumour antigens and/or drug molecules that modulate co-stimulation and cytokine production. These approaches result in anti-tumour effects mediated via generating and/or activating CD4+ and CD8 ${ }^{+} \mathrm{T}$ cells; (2) Nanomedicines can change the polarization of tumour-associated macrophages (TAMs) from a pro-tumour and anti-immunotherapy (M2-like) phenotype into a more anti-tumour and pro-immunotherapy (M1-like) phenotype; (3) Nanomedicines containing chemotherapeutic drugs, such as doxorubicin and oxaliplatin, can boost the induction of immunogenic cell death, which helps reprogram immunogenically 'cold' tumours into 'hot' tumours. Innate immune system-targeted approaches include: (4) Reprogramming tumours into an immunogenically 'hot' phenotype via the activation of PRRs, eliciting a type I interferon response and inducing anti-tumour T cell immunity; and (5) Nanomedicines can be developed to deliver drugs to myeloid cells and their progenitors in the bone marrow, resulting in specific metabolic and epigenetic changes. The ensuing myeloid cells' hyper-responsiveness towards secondary stimuli has become known as 'trained immunity' and may help improve the efficacy of (checkpoint inhibition-based) cancer immunotherapy.

radiotherapy effects can synergize with immune checkpoint blockade-based interventions ${ }^{99}$. In this regard, there is growing evidence that the abscopal effect, whereby local radiotherapy treatment at the primary tumour site can induce regression in distant metastatic lesions, can augment immunotherapy, particularly when combined with smart nanomedicine formulations ${ }^{100}$.

Targeting the innate immune system. Most nano-immunotherapies target the adaptive immune system; modulating components of the innate immune system has thus far remained largely unexplored $^{92,101}$. The innate immune system functions as a rapid and relatively non-specific first line of defence against infections, mediated by the complement system and by cells such as phagocytes and natural killer cells. These cells contain pattern recognition receptors (PRRs), which detect pathogen-associated molecular patterns (PAMPs) and damage-associated molecular patterns (DAMPs). PAMPs and DAMPs can induce an innate immune response and subsequently activate the adaptive immune system ${ }^{92}$. It is becoming increasingly clear that metabolic and epigenetic modifications underlying these responses coordinate a primitive form of innate immune memory referred to as 'trained immunity' ${ }^{102,103}$, which is systemically regulated and preserved by hematopoietic stem cells and progenitor cells in the bone marrow. Accordingly, nanomedicines with bone marrow avidity may help to induce trained immunity to suppress local and metastatic tumour growth ${ }^{92}$.

Alternatively, nanomedicine-based approaches can be designed to induce innate anti-tumour immune responses via activation of PRRs on dendritic cells (DCs) in the TIME, including toll-like receptors (TLRs), retinoic acid-inducible gene I (RIG-I)-like receptors (RLRs) and stimulator of interferon genes (STING). Activating PRRs results in multiple signalling cascades such as transcription of type I interferons (IFN-1). In turn, IFN-1 activates DCs and induces anti-tumour $\mathrm{T}$ cell immunity, providing opportunities to reprogramme immunologically 'cold' tumours into 'hot' tumours 
that are susceptible to checkpoint inhibitor therapy. As an example, cyclodextrin nanoparticles encapsulating the TLR7/8 agonist R848 have been shown to enhance cancer immunotherapy, by promoting the polarization of TAM towards an M1-like phenotype ${ }^{96}$. As a monotherapy, the nanoparticles modulated the immunosuppressive TIME to inhibit tumour growth. The R848-containing formulation furthermore boosted checkpoint inhibitor therapy, in a tumour model unresponsive to anti-PD-1 monotherapy. RLRs are sensor proteins that recognize cytosolic double-stranded RNA. RIG-1 preferentially binds 5'-triphosphorylated RNA and short double-stranded RNA. As such, bispecific siRNAs with 5'-triphosphate ends can be designed to specifically silence an oncogene and to also activate RIG-1. Exploiting this dual strategy, treatment with lipid-coated calcium phosphate nanoparticles containing double-stranded 5'-triphosphorylated anti-Bcl2 siRNA inhibited tumour growth and prolonged survival in mice with orthotopic pancreatic cancer ${ }^{97}$. STING is a key regulatory protein that triggers an IFN-1 response upon detection of cytosolic double-stranded DNA (dsDNA). DsDNA is recognized by cyclic GMP-AMP synthase (cGAS), which induces the production of cyclic guanosine monophosphate-adenosine monophosphate (cGAMP; that is, the high affinity ligand for STING), resulting in a type I interferon response $^{104}$. Nanomedicines are very useful for activating this type of immune response, as cGAMP and other STING agonists are cyclic dinucleotides, and need to be present intracellularly to be recognized by cGAS. As an example, polymersomes designed to enhance cytosolic cGAMP delivery potently inhibited tumour growth and prolonged survival in a mouse melanoma model (alone and in combination with checkpoint inhibitor therapy $)^{105}$.

\section{Outlook}

Improving the clinical impact of cancer nanomedicines requires smart thinking and rational and realistic reasoning. In this perspective, we present four strategic directions to boost cancer nanomedicine performance, translation and exploitation. Like in other areas of oncology drug development, probes and protocols for patient stratification are urgently needed to refine cancer nanomedicine clinical trials. Smart strategies for modular (pro)drug and nanocarrier design as well as library screening will help to maximize the chances that those formulations developed and tested preclinically will eventually perform well in patients. Rationally designed pharmacological and physical combination regimens will amplify the pharmacokinetic and/or pharmacodynamic benefits conferred by entrapping drugs in nanomedicine formulations. Finally, uncovering which pathophysiological features constrain the efficacy of current cancer immunotherapies, and developing immunomodulatory nanomedicines accordingly, will help to improve the outcomes of immuno-oncological interventions and increase the number of long-term survivors.

Beyond the strategies discussed above, several additional directions can be considered to assist in improving the impact of cancer nanomedicine. For instance, the field may profit from implementing guidelines for minimal information reporting to standardize preclinical nanomedicine research and thus promote reproducibility, quantitative comparisons, meta-analyses and modelling ${ }^{106}$. Furthermore, while the majority of nanomedicine formulations are developed for intravenous application, it may be advantageous to also explore other administration routes. For example, certain antibody-based therapeutics, such as Humira (that is, adalimumab; anti-TNF), work well upon subcutaneous self-administration, which provides the advantage of at-home self-administration versus clinical intravenous administration, thereby mitigating the need for hospitalization. Such alternative routes of administration, which have thus far received relatively little consideration in case of nanomedicines, may provide significant advantages in terms of applicability, cost, efficacy and toxicity.
The cancer nanomedicine field has expanded exponentially in recent years. In stark contrast to the numerous new materials and papers that are being produced, only about a dozen nanomedicinal anticancer drugs (antibody-drug conjugates included) have thus far made it to the market. To change this situation, we must move away from continuously making increasingly complex nanomedicine materials and critically reconsider how we are doing translational cancer nanomedicine research. We must establish smart strategies to make nanomedicines work, in as many patients as possible. This shift requires rational and realistic thinking, and integrated and concerted efforts from consortia comprising academics, clinicians, pharmaceutical companies and regulatory authorities. The strategic directions outlined in this manuscript aim to streamline translational cancer nanomedicine research and they will help to promote the clinical impact and patient performance of nanomedicinal anticancer drugs.

Received: 16 April 2019; Accepted: 30 September 2019; Published online: 6 November 2019

\section{References}

1. Peer, D. et al. Nanocarriers as an emerging platform for cancer therapy. Nat Nanotechnol. 2, 751-760 (2007).

2. Shi, J., Kantoff, P. W., Wooster, R. \& Farokhzad, O. C. Cancer nanomedicine: progress, challenges and opportunities. Nat. Rev. Cancer 17, 20-37 (2017).

3. Björnmalm, M., Thurecht, K. J., Michael, M., Scott, A. M. \& Caruso, F Bridging bio-nano science and cancer nanomedicine. ACS Nano 11, 9594-9613 (2017).

4. Van der Meel, R., Lammers, T. \& Hennink, W. E. Cancer nanomedicines: oversold or underappreciated? Expert Opin. Drug Deliv. 14, 1-5 (2017).

5. Anchordoquy, T. J. et al. Mechanisms and barriers in cancer nanomedicine: addressing challenges, looking for solutions. ACS Nano 11, 12-18 (2017).

6. Cobleigh, M. A. et al. Multinational study of the efficacy and safety of humanized anti-HER2 monoclonal antibody in women who have HER2-overexpressing metastatic breast cancer that has progressed after chemotherapy for metastatic disease. J. Clin. Oncol. 17, 2639-48 (1999).

7. Baselga, J. et al. Pertuzumab plus trastuzumab plus docetaxel for metastatic breast cancer. N. Engl. J. Med. 366, 109-119 (2012).

8. Verma, S. et al. Trastuzumab emtansine for HER2-positive advanced breast cancer. N. Engl. J. Med. 367, 1783-1791 (2012).

9. Le, D. T. et al. Mismatch repair deficiency predicts response of solid tumors to PD-1 blockade. Science 357, 409-413 (2017).

10. Autio, K. A. et al. Safety and efficacy of BIND-014, a docetaxel nanoparticle targeting prostate-specific membrane antigen for patients with metastatic castration-resistant prostate cancer: a phase 2 clinical trial. JAMA Oncol. 4, 1344-1351 (2018).

11. Voss, M. H. et al. A randomized phase II trial of CRLX101 in combination with bevacizumab versus standard of care in patients with advanced renal cell carcinoma. Ann. Oncol. 28, 2754-2760 (2017).

12. Fujiwara, Y. et al. A multi-national, randomised, open-label, parallel, phase III non-inferiority study comparing NK105 and paclitaxel in metastatic or recurrent breast cancer patients. Br. J. Cancer 120, 475-480 (2019).

13. Petersen, G. H., Alzghari, S. K., Chee, W., Sankari, S. S. \& La-Beck, N. M. Meta-analysis of clinical and preclinical studies comparing the anticancer efficacy of liposomal versus conventional non-liposomal doxorubicin. J. Control. Release 232, 255-264 (2016).

14. Lammers, T., Rizzo, L. Y., Storm, G. \& Kiessling, F. Personalized nanomedicine. Clin. Cancer Res. 18, 4889-4894 (2012).

15. Bravaccini, S. et al. PSMA expression: a potential ally for the pathologist in prostate cancer diagnosis. Sci. Rep. 8, 1-8 (2018).

16. Han, S., Woo, S., Kim, Y. J. \& Suh, C. H. Impact of 68Ga-PSMA PET on the management of patients with prostate cancer: a systematic review and meta-analysis. Eur. Urol. 74, 179-190 (2018).

17. Kunjachan, S. et al. Passive versus active tumor targeting using RGDand NGR-modified polymeric nanomedicines. Nano Lett. 14, 972-981 (2014).

18. Juweid, M. et al. Micropharmacology of monoclonal antibodies in solid tumors: direct experimental evidence for a binding site barrier. Cancer Res. 52, 5144-53 (1992).

19. Allen, T. M. \& Cullis, P. R. Liposomal drug delivery systems: from concept to clinical applications. Adv. Drug Deliv. Rev. 65, 36-48 (2013).

20. Tsvetkova, Y. et al. Balancing passive and active targeting to different tumor compartments using riboflavin-functionalized polymeric nanocarriers. Nano Lett. 17, 4665-4674 (2017). 
21. Choi, C. H. J., Alabi, C. A., Webster, P. \& Davis, M. E. Mechanism of active targeting in solid tumors with transferrin-containing gold nanoparticles. Proc. Natl Acad. Sci. USA 107, 1235-40 (2010).

22. Kirpotin, D. B. et al. Antibody targeting of long-circulating lipidic nanoparticles does not increase tumor localization but does increase internalization in animal models. Cancer Res. 66, 6732-6740 (2006).

23. Miller, M. A., Arlauckas, S. \& Weissleder, R. Prediction of anti-cancer nanotherapy efficacy by imaging. Nanotheranostics 1, 296-312 (2017).

24. Chen, H., Zhang, W., Zhu, G., Xie, J. \& Chen, X. Rethinking cancer nanotheranostics. Nat. Rev. Mater. 2, 17024 (2017).

25. Miller, M. A. et al. Predicting therapeutic nanomedicine efficacy using a companion magnetic resonance imaging nanoparticle. Sci. Transl. Med. 7, 314 ral83 (2015)

26. Ramanathan, R. K. et al. Correlation between ferumoxytol uptake in tumor lesions by MRI and response to nanoliposomal irinotecan in patients with advanced solid tumors: a pilot study. Clin. Cancer Res. 23, 3638-3648 (2017)

27. Perez-Medina, C. et al. Nanoreporter PET predicts the efficacy of anti-cancer nanotherapy. Nat. Commun. 7, 11838 (2016).

28. Vander Heiden, M. G., Cantley, L. C. \& Thompson, C. B. Understanding the Warburg effect: the metabolic requirements of cell proliferation. Science 324, 1029-33 (2009).

29. Hsu, P. P. \& Sabatini, D. M. Cancer cell metabolism: Warburg and beyond Cell 134, 703-707 (2008).

30. Jørgensen, J. T., Norregaard, K., Martín, M. S., Oddershede, L. B. \& Kjaer, A. Non-invasive early response monitoring of nanoparticle-assisted photothermal cancer therapy using 18F-FDG, 18F-FLT, and 18F-FET PET/ CT imaging. Nanotheranostics 2, 201-210 (2018).

31. Phillips, E. et al. Clinical translation of an ultrasmall inorganic optical-PET imaging nanoparticle probe. Sci. Transl. Med. 6, 260ra149 (2014).

32. Altman, B. J., Stine, Z. E. \& Dang, C. V. From Krebs to clinic: glutamine metabolism to cancer therapy. Nat. Rev. Cancer 16, 619-634 (2016).

33. Garcia-Bermudez, J. et al. Aspartate is a limiting metabolite for cancer cell proliferation under hypoxia and in tumours. Nat. Cell Biol. 20, 775-781 (2018).

34. Knott, S. R. V. et al. Asparagine bioavailability governs metastasis in a model of breast cancer. Nature 554, 378-381 (2018).

35. Lee, $\mathrm{H}$. et al. $64 \mathrm{Cu}-\mathrm{MM}-302$ positron emission tomography quantifies variability of enhanced permeability and retention of nanoparticles in relation to treatment response in patients with metastatic breast cancer. Clin. Cancer Res. 23, 4190-4202 (2017).

36. Edmonds, S. et al. Exploiting the metal-chelating properties of the drug cargo for in vivo positron emission tomography imaging of liposomal nanomedicines. ACS Nano 10, 10294-10307 (2016).

37. Barenholz, Y. Doxil ${ }^{\circledR}$ - the first FDA-approved nano-drug: lessons learned. J. Control. Release 160, 117-134 (2012).

38. Miller, M. A. et al. Tumour-associated macrophages act as a slow-release reservoir of nano-therapeutic Pt(IV) pro-drug. Nat. Commun. 6, 8692 (2015)

39. Karageorgis, A. et al. An MRI-based classification scheme to predict passive access of 5 to 50-nm large nanoparticles to tumors. Sci. Rep. 6, 21417 (2016).

40. Sulheim, E. et al. Multi-modal characterization of vasculature and nanoparticle accumulation in five tumor xenograft models. J. Control. Release 279, 292-305 (2018)

41. Theek, B. et al. Histidine-rich glycoprotein-induced vascular normalization improves EPR-mediated drug targeting to and into tumors. J. Control. Release 282, 25-34 (2018).

42. Hare, J. I. et al. Challenges and strategies in anti-cancer nanomedicine development: an industry perspective. Adv. Drug Deliv. Rev. 108, 25-38 (2017)

43. Szebeni, J., Simberg, D., González-Fernández, Á., Barenholz, Y. \& Dobrovolskaia, M. A. Roadmap and strategy for overcoming infusion reactions to nanomedicines. Nat. Nanotechnol. 13, 1100-1108 (2018).

44. Qi, R. et al. Nanoparticle conjugates of a highly potent toxin enhance safety and circumvent platinum resistance in ovarian cancer. Nat. Commun. 8, 2166 (2017).

45. Ashton, S. et al. Aurora kinase inhibitor nanoparticles target tumors with favorable therapeutic index in vivo. Sci. Transl. Med. 8, 325ral7 (2016).

46. Adams, D. et al. Patisiran, an RNAi therapeutic, for hereditary transthyretin amyloidosis. N. Engl. J. Med. 379, 11-21 (2018)

47. Sahin, U. \& Türeci, Ö. Personalized vaccines for cancer immunotherapy. Science 359, 1355-1360 (2018).

48. Pardi, N., Hogan, M. J., Porter, F. W. \& Weissman, D. mRNA vaccines a new era in vaccinology. Nat. Rev. Drug Discov. 17, 261-279 (2018).

49. Pardi, N. et al. Administration of nucleoside-modified mRNA encoding broadly neutralizing antibody protects humanized mice from HIV-1 challenge. Nat. Commun. 8, 14630 (2017).

50. Kranz, L. M. et al. Systemic RNA delivery to dendritic cells exploits antiviral defence for cancer immunotherapy. Nature 534, 396-401 (2016).

51. Kreiter, S. et al. Mutant MHC class II epitopes drive therapeutic immune responses to cancer. Nature 520, 692-696 (2015).
52. Oberli, M. A. et al. Lipid nanoparticle assisted mRNA delivery for potent cancer immunotherapy. Nano Lett. 17, 1326-1335 (2017).

53. Sahin, U. et al. Personalized RNA mutanome vaccines mobilize poly-specific therapeutic immunity against cancer. Nature 547, 222-226 (2017).

54. Zhang, Y., Li, N., Suh, H. \& Irvine, D. J. Nanoparticle anchoring targets immune agonists to tumors enabling anti-cancer immunity without systemic toxicity. Nat. Commun. 9, 6 (2018).

55. Charych, D. H. et al. NKTR-214, an engineered cytokine with biased IL2 receptor binding, increased tumor exposure, and marked efficacy in mouse tumor models. Clin. Cancer Res. 22, 680-690 (2016).

56. Bentebibel, S.-E. et al. A first-in-human study and biomarker analysis of NKTR-214, a novel IL2R $\beta \gamma$-biased cytokine, in patients with advanced or metastatic solid tumors. Cancer Discov. 9, 711-721 (2019).

57. Cern, A., Marcus, D., Tropsha, A., Barenholz, Y. \& Goldblum, A. New drug candidates for liposomal delivery identified by computer modeling of liposomes' remote loading and leakage. J. Control. Release 252, 18-27 (2017).

58. Zhao, Y. et al. Augmenting drug-carrier compatibility improves tumour nanotherapy efficacy. Nat. Commun. 7, 11221 (2016).

59. $\mathrm{Hu}, \mathrm{Q}$. et al. Complete regression of breast tumour with a single dose of docetaxel-entrapped core-cross-linked polymeric micelles. Biomaterials 53, 370-378 (2015).

60. Cristal Therapeutics. Efficacy study of CPC634 (CriPec ${ }^{\circledast}$ docetaxel) in platinum resistant ovarian cancer (CINOVA) ClinicalTrials.gov https://clinicaltrials.gov/ct2/show/NCT03742713 (2018).

61. Wang, $H$. et al. New generation nanomedicines constructed from self-assembling small-molecule prodrugs alleviate cancer drug toxicity. Cancer Res. 77, 6963-6974 (2017).

62. Miller, M. A. et al. Modular nanoparticulate prodrug design enables efficient treatment of solid tumors using bioorthogonal activation. ACS Nano 12, 12814-12826 (2018)

63. Hrkach, J. et al. Preclinical development and clinical translation of a PSMA-targeted docetaxel nanoparticle with a differentiated pharmacological profile. Sci. Transl. Med. 4, 128ra39 (2012).

64. Wang, E. T. et al. Barcoded nanoparticles for high throughput in vivo discovery of targeted therapeutics. Proc. Natl Acad. Sci. USA 114, 2060-2065 (2017)

65. Sago, C. D. et al. Nanoparticles that deliver RNA to bone marrow identified by in vivo directed evolution. J. Am. Chem. Soc. 140, 17095-17105 (2018).

66. Yaari, Z. et al. Theranostic barcoded nanoparticles for personalized cancer medicine. Nat. Commun. 7, 13325 (2016).

67. Wang-Gillam, A. et al. Nanoliposomal irinotecan with fluorouracil and folinic acid in metastatic pancreatic cancer after previous gemcitabine-based therapy (NAPOLI-1): a global, randomised, open-label, phase 3 trial. Lancet 387, 545-557 (2016)

68. Chen, L. T. et al. Final results of NAPOLI-1: a phase 3 study of nal-IRI (MM-398) \pm 5 -fluorouracil and leucovorin (5-FU/LV) vs 5 -FU/LV in metastatic pancreatic cancer (mPAC) previously treated with gemcitabinebased therapy. Ann. Oncol. 27, 622PD-622PD (2016).

69. Schmid, P. et al. Atezolizumab and nab-paclitaxel in advanced triplenegative breast cancer. N. Engl. J. Med. 379, 2108-2121 (2018).

70. Chauhan, V. P. et al. Angiotensin inhibition enhances drug delivery and potentiates chemotherapy by decompressing tumour blood vessels. Nat. Commun. 4, 2516 (2013).

71. Diop-Frimpong, B., Chauhan, V. P., Krane, S., Boucher, Y. \& Jain, R. K. Losartan inhibits collagen I synthesis and improves the distribution and efficacy of nanotherapeutics in tumors. Proc. Natl Acad. Sci. USA 108, 2909-2914 (2011).

72. Murphy, J. E. et al. Potentially curative combination of TGF-b1 inhibitor losartan and FOLFIRINOX (FFX) for locally advanced pancreatic cancer (LAPC): R0 resection rates and preliminary survival data from a prospective phase II study. J. Clin. Oncol. 36, 4116 (2018).

73. Murphy, J. E. et al. Total neoadjuvant therapy with FOLFIRINOX in combination with losartan followed by chemoradiotherapy for locally advanced pancreatic cancer. JAMA Oncol. 5, 1020 (2019).

74. Abumanhal-Masarweh, $\mathrm{H}$. et al. Sodium bicarbonate nanoparticles modulate the tumor $\mathrm{pH}$ and enhance the cellular uptake of doxorubicin. J. Control. Release 296, 1-13 (2019).

75. Lancet, J. E. et al. CPX-351 (cytarabine and daunorubicin) liposome for injection versus conventional cytarabine plus daunorubicin in older patients with newly diagnosed secondary acute myeloid leukemia. J. Clin. Oncol. 36, 2684-2692 (2018)

76. Davies, C. D. L. et al. Radiation improves the distribution and uptake of liposomal doxorubicin (Caelyx) in human osteosarcoma xenografts. Cancer Res. 64, 547-553 (2004).

77. Lammers, T. et al. Image-guided and passively tumour-targeted polymeric nanomedicines for radiochemotherapy. Br. J. Cancer 99, 900-910 (2008).

78. Miller, M. A. et al. Radiation therapy primes tumors for nanotherapeutic delivery via macrophage-mediated vascular bursts. Sci. Transl. Med. 9, eaal0225 (2017). 
79. Min, Y. et al. Antigen-capturing nanoparticles improve the abscopal effect and cancer immunotherapy. Nat. Nanotechnol. 12, 877-882 (2017).

80. Snipstad, S. et al. Sonopermeation to improve drug delivery to tumors: from fundamental understanding to clinical translation. Expert Opin. Drug Deliv. 15, 1249-1261 (2018).

81. Dimcevski, G. et al. A human clinical trial using ultrasound and microbubbles to enhance gemcitabine treatment of inoperable pancreatic cancer. J. Control. Release 243, 172-181 (2016).

82. Carpentier, A. et al. Clinical trial of blood-brain barrier disruption by pulsed ultrasound. Sci. Transl. Med. 8, 343re2 (2016).

83. Mainprize, T. et al. Blood-brain barrier opening in primary brain tumors with non-invasive MR-guided focused ultrasound: a clinical safety and feasibility study. Sci. Rep. 9, 321 (2019).

84. Tak, W. Y. et al. Phase III HEAT study adding lyso-thermosensitive liposomal doxorubicin to radiofrequency ablation in patients with unresectable hepatocellular carcinoma lesions. Clin. Cancer Res. 24, 73-83 (2018).

85. Celsion. Study of ThermoDox with standardized radiofrequency ablation (RFA) for treatment of hepatocellular carcinoma (HCC) (OPTIMA). ClinicalTrial.gov https://clinicaltrials.gov/ct2/show/NCT02112656 (2014).

86. Kim, A. A phase I study of lyso-thermosensitive liposomal doxorubicin and MR-HIFU for pediatric refractory solid tumors. ClinicalTrial.gov https://clinicaltrials.gov/ct2/show/NCT02536183?term=thermodox\&rank $=10$ (2015).

87. Ribas, A. \& Wolchok, J. D. Cancer immunotherapy using checkpoint blockade. Science 359, 1350-1355 (2018).

88. June, C. H. et al. CAR T cell immunotherapy for human cancer. Science 359, 1361-1365 (2018).

89. Jain, R. K. et al. Understanding the tumor immune microenvironment (TIME) for effective therapy. Nat. Med. 24, 541-550 (2018).

90. Riley, R. S., June, C. H., Langer, R. \& Mitchell, M. J. Delivery technologies for cancer immunotherapy. Nature Reviews Drug Discovery 18, 175-196 (2019).

91. Sun, Q. et al. Nanomedicine and macroscale materials in immunooncology. Chem. Soc. Rev. 48, 351-381 (2019).

92. Mulder, W. J. M., Ochando, J., Joosten, L. A. B., Fayad, Z. A. \& Netea, M. G. Therapeutic targeting of trained immunity. Nat. Rev. Drug Discov. 18, 553-566 (2019).

93. Jiang, W. et al. Designing nanomedicine for immuno-oncology. Nat. Biomed. Eng. 1, 0029 (2017).

94. Friedman, C. F., Proverbs-Singh, T. A. \& Postow, M. A. Treatment of the immune-related adverse effects of immune checkpoint inhibitors: a review. JAMA Oncol. 2, 1346-1353 (2016).

95. Pfirschke, C. et al. Immunogenic chemotherapy sensitizes tumors to checkpoint blockade therapy. Immunity 44, 343-354 (2016).

96. Prendergast, G. C., Malachowski, W. P., DuHadaway, J. B. \& Muller, A. J. Discovery of IDO1 inhibitors: from bench to bedside. Cancer Res. 77, 6795-6811 (2017).

97. Lu, J. et al. Nano-enabled pancreas cancer immunotherapy using immunogenic cell death and reversing immunosuppression. Nat. Commun. 8, 1811 (2017).
98. Lu, J. et al. Breast cancer chemo-immunotherapy through liposomal delivery of an immunogenic cell death stimulus plus interference in the IDO-1 pathway. ACS Nano 12, 11041-11061 (2018).

99. Hwang, W. L., Pike, L. R. G., Royce, T. J., Mahal, B. A. \& Loeffler, J. S. Safety of combining radiotherapy with immune-checkpoint inhibition. Nat. Rev. Clin. Oncol. 15, 477-494 (2018).

100. Ngwa, W. et al. Using immunotherapy to boost the abscopal effect. Nat. Rev. Cancer 18, 313-322 (2018).

101. Borden, E. C. Interferons $\alpha$ and $\beta$ in cancer: therapeutic opportunities from new insights. Nat. Rev. Drug Discov. 18, 219-234 (2019).

102. Netea, M. G., Latz, E., Mills, K. H. G. \& O’Neill, L. A. J. Innate immune memory: a paradigm shift in understanding host defense. Nat. Immunol. 16, 675-679 (2015).

103. Netea, M. G. et al. Trained immunity: A program of innate immune memory in health and disease. Science 352, aaf1098 (2016).

104. Chen, Q., Sun, L. \& Chen, Z. J. Regulation and function of the cGAS-STING pathway of cytosolic DNA sensing. Nat. Immunol. 17, 1142-1149 (2016)

105. Shae, D. et al. Endosomolytic polymersomes increase the activity of cyclic dinucleotide STING agonists to enhance cancer immunotherapy. Nat. Nanotechnol. 14, 269-278 (2019).

106. Faria, M. et al. Minimum information reporting in bio-nano experimental literature. Nat. Nanotechnol. 13, 777-785 (2018).

\section{Acknowledgements}

R.v.d.M. is supported by the Netherlands Organization for Scientific Research (NWO; Veni STW grant no. 14385). E.S. is supported by the Central Norway Regional Health Authority (no. 46084000). Y.S. and T.L. acknowledge support by the European Union (European Fund for Regional Development: I3-STM (no. 0800387)). F.K. and T.L. are supported by the German Research Foundation (DFG; GRK 2375 (no. 331065168 )). W.J.M.M. is supported by the National Institutes of Health (R01 HL118440, R01 HL125703, P01 HL131478) and by the Netherlands Organization for Scientific Research (NWO; ZonMW Vici grant no. 016.176.622). T.L. is supported by the European Research Council (no. 309495, no. 680882 and no. 813086), the European Commission (ERA-NET: NSC4DIPG), the German Research Foundation (DFG; SFB1066, SFB/TRR57) and the Aachen Interdisciplinary Center for Clinical Research (IZKF; no. O3-2).

\section{Competing interests}

The authors declare no competing interests.

\section{Additional information}

\section{Correspondence should be addressed to T.L.}

Reprints and permissions information is available at www.nature.com/reprints. Publisher's note Springer Nature remains neutral with regard to jurisdictional claims in published maps and institutional affiliations.

(c) Springer Nature Limited 2019 